\title{
Instantons on Special Holonomy Manifolds
}

\author{
Tatiana A. Ivanova and Alexander D. Popov \\ Bogoliubov Laboratory of Theoretical Physics, JINR \\ 141980 Dubna, Moscow Region, Russia \\ Email: ita, popov@theor.jinr.ru
}

\begin{abstract}
We consider cones over manifolds admitting real Killing spinors and instanton equations on connections on vector bundles over these manifolds. Such cones are manifolds with special (reduced) holonomy. We generalize the scalar ansatz for a connection proposed by Harland and Nölle [1] in such a way that instantons are parameterized by constrained matrix-valued functions. Our ansatz reduces instanton equations to a matrix model equations which can be further reduced to Newtonian mechanics with particle trajectories obeying first-order gradient flow equations. Generalizations to Kähler-Einstein manifolds and resolved Calabi-Yau cones are briefly discussed. Our construction allows one to associate quiver gauge theories with special holonomy manifolds via dimensional reduction.
\end{abstract}




\section{Introduction}

Instantons in four dimensions [2] are nonperturbative Bogomolny-Prasad-Sommerfeld (BPS) configurations solving first-order anti-self-duality equations for gauge fields which imply the full YangMills equations. They are important objects in modern field theory [3, 4]. Generalization of YangMills instantons to higher dimensions, proposed in [5] and studied in [6]-[12] (for more literature see references therein), is important both in mathematics [10,11] and string theory [13, 14]. Some of their solutions on spaces $\mathbb{R}^{n}$ with $n>5$ were obtained in $[6,15,16]$. Constructions of solutions to the instanton equations on more general curved homogeneous manifolds as well as on cylinders and cones over them were considered in $[17,18]$. The construction on coset spaces, many of which admit Killing spinors [19], was generalized to cones over manifolds with real Killing spinors [1], not necessarily homogeneous (see also [20] about instantons on Calabi-Yau cones and their resolutions). All these solutions were lifted to solutions of heterotic supergravity equations supplemented by the Bianchi identity [21, 22, 20, 1, 23].

Riemannian manifolds $\left(M, g_{M}\right)$ with real Killing spinors ${ }^{1}$ often occur in string theory compactifications (see e.g. $[1,21,22,23]$ and references therein). These manifolds were classified in [19]. Besides the round spheres they are

- nearly Kähler 6-manifolds $M$, SU(3)-structure

- nearly parallel 7-manifolds $M, G_{2}$-structure

- Sasaki-Einstein $(2 m+1)$-manifolds $M, \mathrm{SU}(m)$-structure

- 3-Sasakian $(4 m+3)$-manifolds $M, \operatorname{Sp}(m)$-structure

All these manifolds have a connection with a non-vanishing torsion and admit a non-integrable $H$-structure mentioned above, i.e. a reduction of the structure group $\mathrm{SO}(n)$ of the tangent bundle $T M$ to $H \subset \mathrm{SO}(n)$. The above manifolds are equipped with canonical 3 -form $P$ and 4 -form $Q$ defined via the Killing spinors.

Recall that instanton equations on an $(n+1)$-dimensional Riemannian manifold $X$ can be introduced as follows. Suppose there exists a 4 -form $Q$ on $X$. Then there exists an $(n-3)$-form $* Q$, where $*$ is the Hodge operator on $X$. Let $\mathcal{A}$ be a connection on a bundle over $X$ with the curvature $\mathcal{F}$. Then the generalized anti-self-duality equation on the gauge field $\mathcal{F}$ is $[10,11]$

$$
* \mathcal{F}+* Q \wedge \mathcal{F}=0
$$

For $n+1>4$ these equations can be defined on manifolds $X$ with special holonomy, i.e. such that the holonomy group $G$ of the Levi-Civita connection on the tangent bundle $T X$ is a subgroup in the group $\mathrm{SO}(n+1)$. On such manifolds any solution of eq.(1.1) satisfies to the Yang-Mills equation. The instanton equation (1.1) is also well-defined on manifolds $X$ with non-integrable $G$ structures but then (1.1) implies the Yang-Mills equation with torsion. This torsion term vanishes on manifolds with real Killing spinors [1].

\footnotetext{
${ }^{1} \mathrm{~A}$ Killing spinor on a Riemannian manifold $N$ is a spinor field $\epsilon$ which satisfies $\nabla_{L} \epsilon=\mathrm{i} \lambda L \cdot \epsilon$ for all tangent vectors $L$, where $\nabla$ is the spinor covariant derivative, $\cdot$ is Clifford multiplication and $\lambda$ is a constant. If $\lambda=0$ then the spinor is called parallel, and $N$ is a manifold with special (reduced) holonomy.
} 
In this paper, we mostly consider $X=\mathcal{C}(M)$, where $M$ is a manifold with real Killing spinors and $\mathcal{C}(M)$ is a cone over $M$ with the metric

$$
g_{X}=\mathrm{d} r^{2}+r^{2} g_{M}=e^{2 \tau}\left(\mathrm{d} \tau^{2}+g_{M}\right) \quad \text { for } \quad r:=e^{\tau} .
$$

From (1.2) it follows that the cone $\mathcal{C}(M)$ is conformally equivalent to the cylinder

$$
Z=\mathbb{R} \times M
$$

with the metric

$$
g_{Z}=\mathrm{d} \tau^{2}+g_{M} .
$$

Furthermore, one can show [1] that the equation (1.1) on the cone $X=\mathcal{C}(M)$ is related with instanton equation on the cylinder $Z=\mathbb{R} \times M$ as follows

$$
*_{X} \mathcal{F}+*_{X} Q_{X} \wedge \mathcal{F}=e^{(n-3) \tau}\left(*_{Z} \mathcal{F}+*_{Z} Q_{Z} \wedge \mathcal{F}\right)=0,
$$

where $n+1=\operatorname{dim} \mathcal{C}(M)=\operatorname{dim} Z$. In other words, eq.(1.1) on $\mathcal{C}(M)$ is equivalent to the equation on $\mathbb{R} \times M$ after rescaling (1.2) of the metric. That is why in the following we will consider the instanton equation

$$
* \mathcal{F}+* Q_{Z} \wedge \mathcal{F}=0
$$

on the cylinder $Z$ over $M$. Here we omit the index $Z$ in the star operator. Note that components of $\mathcal{F}$ on the cone can be obtained from those on the cylinder simply via rescaling (1.2).

In this paper, we generalize the results [1] of Harland and Nölle on investigating instantons on cones over manifolds with Killing spinors. First, in section 2, we collect various facts concerning nearly Kähler, nearly parallel $G_{2}$, Sasaki-Einstein and 3-Sasakian manifolds $M$ following mainly the description in [1]. We describe metrics on $M$, canonical connections and various $q$-forms $(q=1,2, \ldots)$ as well as their extension to the cylinder $Z=\mathbb{R} \times M$. Then, in section 3 , we introduce an ansatz for a gauge potential $\mathcal{A}$ which reduces the instanton equation (1.6) on $\mathbb{R} \times M$ to a matrix equations on $\mathbb{R}$. Resolution of natural algebraic constraints on the matrices yields further reduction to a set of first-order equations on functions depending on $\tau \in \mathbb{R}$. These equations are gradient flow equations describing BPS-type trajectories in Newtonian mechanics of particles moving in $\mathbb{R}^{N}$, where $N$ is the number of functions parameterizing matrices in the ansatz for a gauge potential $\mathcal{A}$. Solutions to these equations give instanton solutions of the Yang-Mills equations on $\mathbb{R} \times M$ and their extension to the cone $\mathcal{C}(M)$. Finally, in section 4, we discuss some generalizations of our construction allowing one to associate quiver gauge theories with such special holonomy manifolds as Kähler-Einstein manifolds and resolved Calabi-Yau cones.

\section{Manifolds with Killing spinors}

\subsection{Nearly Kähler 6-manifolds}

Consider the cylinder (1.3) with the metric (1.4), where $M$ is a nearly Kähler 6-manifold. It is defined as a manifold with a 2 -form $\omega$ and a 3 -form $P$ such that

$$
\mathrm{d} \omega=3 *_{M} P \quad \text { and } \quad \mathrm{d} P=2 \omega \wedge \omega=: 4 Q .
$$


For a local orthonormal co-frame $\left\{e^{a}\right\}$ on $M$ one can choose

$$
\omega=e^{12}+e^{34}+e^{56} \quad \text { and } \quad P=e^{135}+e^{164}-e^{236}-e^{245},
$$

where $a=1, \ldots, 6, e^{a_{1} \ldots a_{l}}:=e^{1} \wedge \ldots \wedge e^{l}$, and get

$$
*_{M} P=e^{145}+e^{235}+e^{136}-e^{246}, \quad Q=e^{1234}+e^{1256}+e^{3456} .
$$

Here $*_{M}$ denotes the Hodge operator on $M$. On $Z$ one can introduce the 4 -form

$$
Q_{Z}=\mathrm{d} \tau \wedge P+Q
$$

which is used in the instanton equation (1.6).

The canonical connection $\tilde{\Gamma}$ on $M$, which is a metric-compatible connection with totally antisymmetric (intrinsic) torsion, has $\mathrm{SU}(3)$ structure group. This connection has components

$$
\tilde{\Gamma}_{a b}^{c}=\Gamma_{a b}^{c}+\frac{1}{2} P_{c a b}
$$

where $\Gamma_{a b}^{c}$ are components of the Levi-Civita connection and

$$
P_{a b c}=T_{b c}^{a}
$$

are components of the torsion $T^{a}=\frac{1}{2} T_{b c}^{a} e^{b} \wedge e^{c}$ defined from the Cartan structure equations

$$
\mathrm{d} e^{a}+\tilde{\Gamma}_{b}^{a} \wedge e^{b}=T^{a}
$$

for basis 1-forms $e^{a}$.

Note that the structure group of $M$ is $\mathrm{SU}(3)$ (or its subgroup) and $P$ induces a $G_{2}$-structure on $Z$ since $\mathrm{SU}(3) \subset G_{2}$. Recall that $\mathfrak{g}_{2}=\mathfrak{s u}(3) \oplus \mathfrak{m}$, $\operatorname{dim} \mathfrak{m}=6$, and one can define the generators of $G_{2}$ as $7 \times 7$ matrices from $\mathfrak{s o}(7)$ with the commutation relations

$$
\left[I_{i}, I_{j}\right]=f_{i j}^{k} I_{k}, \quad\left[I_{i}, I_{a}\right]=f_{i a}^{b} I_{b} \quad \text { and } \quad\left[I_{a}, I_{b}\right]=f_{a b}^{i} I_{i}+f_{a b}^{c} I_{c}
$$

where $I_{i} \in \mathfrak{s u}(3), I_{a} \in \mathfrak{m}$ and $f$ 's are structure constants of $\mathfrak{g}_{2}$. One can choose [1]

$$
\begin{array}{ll}
I_{i a}^{b}=f_{i a}^{b}, & I_{i a}^{0}=-I_{i 0}^{a}=0, \\
I_{a b}^{c}=\frac{1}{2} f_{a b}^{c}, & I_{a 0}^{b}=-I_{a b}^{0}=\delta_{a}^{b},
\end{array}
$$

and obtain

$$
P_{a b c}=-f_{a b}^{c} .
$$

Introducing $\mu=(0, a)$, we can denote matrices in (2.9) as $I_{i \nu}^{\mu}$ and $I_{a \nu}^{\mu}$. The extension of the canonical connection $\tilde{\Gamma}$ to $Z$ has the same components $(2.5)$ and can be written as

$$
\tilde{\Gamma}=\tilde{\Gamma}^{i} I_{i}
$$

with $I_{i}$ given in $(2.9)$. 


\section{$2.2 \quad$ Nearly parallel $G_{2}$-manifolds}

Let us consider the cylinder (1.3) over a nearly parallel $G_{2}$-manifold $M$. It is defined as a manifold with a 3 -form $P$ (a $G_{2}$-structure) preserved by the group $G_{2} \subset \mathrm{SO}(7)$ such that

$$
\mathrm{d} P=\gamma *_{M} P
$$

for some constant $\gamma \in \mathbb{R}$. For a local orthonormal co-frame $e^{a}, a=1, \ldots, 7$, on $M$ one can choose

$$
P=e^{123}+e^{145}-e^{167}+e^{246}+e^{257}+e^{347}-e^{356}
$$

and therefore

$$
*_{M} P=: Q=e^{4567}+e^{2367}-e^{2345}+e^{1357}+e^{1346}+e^{1256}-e^{1247} .
$$

It is easy to see that for the choice $(2.13)$ one obtains $\mathrm{d} P=4 Q$, i.e. $\gamma=4$. The 4 -form $Q_{Z}$ on $Z$ can be chosen similar to $(2.4)$ as

$$
Q_{Z}=\mathrm{d} \tau \wedge P+Q
$$

This form defines a $\operatorname{Spin}(7)$-structure on $Z$.

One can define generators of the group Spin(7) via the structure constants $f_{i j}^{k}, f_{i a}^{b}$ and $f_{a b}^{c}$ of the group $\operatorname{Spin}(7)$ by using the decomposition $\mathfrak{s p i n}(7)=\mathfrak{g}_{2} \oplus \mathfrak{m}$, as $8 \times 8$ matrices $I_{i}=\left(I_{i \nu}^{\mu}\right) \in \mathfrak{g}_{2}$ and $I_{a}=\left(I_{a \nu}^{\mu}\right) \in \mathfrak{m}, \operatorname{dim} \mathfrak{m}=7, \mu=(0, a)$. The generators $I_{i}, I_{a}$ have the same form as in $(2.9)$ but with structure constants $f$ 's of $\operatorname{Spin}(7)$.

The canonical connection $\tilde{\Gamma}$ on $M$ is not changed after its extension to $Z$ and has the components

$$
\tilde{\Gamma}=\tilde{\Gamma}^{i} I_{i} \quad \Rightarrow \quad \tilde{\Gamma}_{a}^{i} I_{i b}^{c}=\tilde{\Gamma}_{a b}^{c}=\Gamma_{a b}^{c}+\frac{1}{3} P_{a b c}
$$

with torsion components

$$
T_{b c}^{a}=\frac{2}{3} P_{a b c} .
$$

\subsection{Sasaki-Einstein manifolds}

Consider now the cylinder (1.3) with the metric (1.4), where $M$ is a Sasaki-Einstein manifold. It is a $(2 m+1)$-dimensional manifold such that the cone $\mathcal{C}(M)$ with the metric (1.2) is a CalabiYau $(m+1)$-fold [24]. Such manifolds $M$ have the structure group $\mathrm{SU}(m) \subset \mathrm{SO}(2 m+1)$ and the holonomy group of $\mathcal{C}(M)$ is $\mathrm{SU}(m+1)$. Sasaki-Einstein manifolds are endowed with 1-, 2-, 3- and 4 -forms $\eta, \omega, P$ and $Q$, which can be defined in an orthonormal basis $e^{1}, e^{a}, a=2, \ldots, 2 m+1$, as

$$
\eta=e^{1}, \quad \omega=e^{23}+e^{45}+\cdots+e^{2 m 2 m+1}, \quad P=\eta \wedge \omega \quad \text { and } \quad Q=\frac{1}{2} \omega \wedge \omega .
$$

One can check that $\eta\lrcorner \omega=0$ and

$$
\mathrm{d} \eta=2 \omega, \quad \mathrm{d} *_{M} \omega=2 m *_{M} \eta, \quad \mathrm{d} P=4 Q \quad \text { and } \quad \mathrm{d} *_{M} Q=(2 m-2) *_{M} P .
$$

The metric on $Z$ has the form (1.4) with

$$
g_{M}=\left(e^{1}\right)^{2}+\exp (2 h) \delta_{a b} e^{a} e^{b} .
$$


Note that for the value of $h$ such that

$$
\exp (2 h)=\frac{2 m}{m+1}
$$

the torsion of the canonical connection on $M$ (and on $Z$ ) becomes antisymmetric [1], but we keep the one-parameter family (2.20) of Sasakian metric including the case $h=0$ when the metric is Einstein. Components of the canonical connection $\tilde{\Gamma}$ are

$$
\tilde{\Gamma}_{\mu a}^{b}=\Gamma_{\mu a}^{b}+\frac{1}{m} P_{\mu a b}, \quad-\tilde{\Gamma}_{\mu a}^{1}=\tilde{\Gamma}_{\mu 1}^{a}=\Gamma_{\mu 1}^{a}+P_{\mu 1 a},
$$

where $\mu=(1, a)$ and the torsion of $\tilde{\Gamma}$ is

$$
T^{1}=P_{1 \mu \nu} e^{\mu} \wedge e^{\nu} \quad \text { and } \quad T^{a}=\frac{m+1}{2 m} P_{a \mu \nu} e^{\mu} \wedge e^{\nu} .
$$

As 4 -form $Q_{Z}$ on $Z$ one can take [1]

$$
Q_{Z}=\exp (2 h) \mathrm{d} \tau \wedge P+\exp (4 h) Q,
$$

where $P$ and $Q$ are given in (2.18).

Let $\hat{\mu}=(0, \mu)=(0,1, a)$. Then one can define generators of the group $\mathfrak{s u}(m+1)=\mathfrak{s u}(m) \oplus \mathfrak{m}$ as $(2 m+1) \times(2 m+1)$ antisymmetric matrices $I_{i}=\left(I_{i} \widehat{\widehat{\mu}}\right) \in \mathfrak{s u}(m)$ and $I_{\mu}=\left(I_{\mu} \widehat{\widehat{\nu}}\right) \in \mathfrak{m}$ such that non-vanishing components are

$$
\begin{gathered}
I_{i a}^{b}=f_{i a}^{b}, \\
I_{1 a}^{b}=-\frac{1}{m} P_{1 a b}=(m+1) f_{1 a}^{b}, \quad-I_{a b}^{0}=I_{a 0}^{b}=\delta_{a}^{b}, \\
I_{a b}^{1}=-I_{a 1}^{b}=-P_{1 a b}=\frac{1}{2} f_{a b}^{1},
\end{gathered}
$$

where $f_{i a}^{b}, f_{a b}^{1}$ and $f_{1 a}^{b}$ are parts of the structure constants of $\mathfrak{s u}(m+1)$. In terms of these matrices the canonical connection $\tilde{\Gamma}$ on $M$ pulled-back to $Z$ can be written as

$$
\tilde{\Gamma}=\tilde{\Gamma}^{i} I_{i}=e^{\mu} \tilde{\Gamma}_{\mu}^{i} I_{i}
$$

\subsection{3-Sasakian manifolds}

Let us now consider the cylinder (1.3) over a 3-Sasakian manifold $M$. It is defined as a $(4 m+3)$ dimensional manifold such that the cone $\mathcal{C}(M)$ over it is a hyper-Kähler $(4 m+4)$-manifold [24], i.e. the holonomy group of $\mathcal{C}(M)$ is $\operatorname{Sp}(m+1)$. The structure group of $M$ is $\operatorname{Sp}(m)$ and any 3-Sasakian manifold can be endowed with three 1-forms $\eta^{\alpha}$, three 2-forms $\omega^{\alpha}$, a 3-form $P$ and a 4 -form $Q$, $\alpha=1,2,3$ [24]. In a local orthonormal co-frame $e^{\alpha}, e^{a}, a=4, \ldots, 4 m+3$, these forms can be written as

$$
\begin{aligned}
& \eta^{1}=e^{1}, \quad \omega^{1}=e^{45}+e^{67}+\cdots+e^{4 m 4 m+1}+e^{4 m+24 m+3}, \\
& \eta^{2}=e^{2}, \quad \omega^{2}=e^{46}-e^{57}+\cdots+e^{4 m 4 m+2}-e^{4 m+14 m+3}, \\
& \eta^{3}=e^{3}, \quad \omega^{3}=e^{47}+e^{56}+\cdots+e^{4 m 4 m+3}+e^{4 m+14 m+2}, \\
& P=\frac{1}{3} \eta^{123}+\frac{1}{3} \eta^{\alpha} \wedge \omega^{\alpha} \quad \text { and } \quad Q=\frac{1}{6} \omega^{\alpha} \wedge \omega^{\alpha} .
\end{aligned}
$$


The forms $\eta^{\alpha}$ and $\omega^{\alpha}$ satisfy the equations

$$
\mathrm{d} \eta^{\alpha}=\varepsilon_{\alpha \beta \gamma} \eta^{\beta} \wedge \eta^{\gamma}+2 \omega^{\alpha}, \quad \mathrm{d} \omega^{\alpha}=2 \varepsilon_{\alpha \beta \gamma} \eta^{\beta} \wedge \omega^{\gamma} .
$$

We introduce indices $\mu=(\alpha, a)$ and $\widehat{\mu}=(0, \mu)=(0, \alpha, a)$. Using the splitting

$$
\mathfrak{s p}(m+1)=\mathfrak{s p}(m) \oplus \mathfrak{m}, \quad \operatorname{dim} \mathfrak{m}=4 m+3,
$$

one can introduce generators $I_{i}=\left(I_{i} \widehat{\mu}\right) \in \mathfrak{s p}(m)$ and $I_{a}=\left(I_{a}^{\widehat{\mu}}\right) \in \mathfrak{m}$ of the group $\operatorname{Sp}(m+1)$ as matrices from $\mathfrak{s o}(4 m+4)$. One can take them so that non-vanishing components are [1]

$$
\begin{gathered}
I_{i a}^{b}=f_{i a}^{b}, \\
I_{\alpha \beta}^{\gamma}=-\varepsilon_{\alpha \beta \gamma}=-3 P_{\alpha \beta \gamma}=\frac{1}{2} f_{\alpha \beta}^{\gamma}, \quad I_{\alpha 0}^{\beta}=\delta_{\alpha}^{\beta}, \\
I_{a b}^{\alpha}=-\omega_{a b}^{\beta}=-3 P_{\alpha a b}=\frac{1}{2} f_{a b}^{\alpha}, \quad I_{a 0}^{b}=\delta_{a}^{b},
\end{gathered}
$$

where $f$ 's are the structure constants of the group $\operatorname{Sp}(m+1)$.

Note that the metric on $Z=\mathbb{R} \times M$ has the form (1.4) with a one-parameter family

$$
g_{M}=\delta_{\alpha \beta} e^{\alpha} e^{\beta}+\exp (2 h) \delta_{a b} e^{a} e^{b}
$$

of metrics on $M$. The 4 -form $Q_{Z}$ can be chosen as

$$
Q_{Z}=\frac{1}{6}\left(\exp (4 h) \omega^{\alpha} \wedge \omega^{\alpha}+\exp (2 h) \varepsilon_{\alpha \beta \gamma} \omega^{\alpha} \wedge \eta^{\beta} \wedge \eta^{\gamma}+2 \exp (2 h) \mathrm{d} \tau \wedge \eta^{\alpha} \wedge \omega^{\alpha}+6 \mathrm{~d} \tau \wedge \eta^{123}\right) .
$$

In terms of the matrices (2.30) the canonical connection $\tilde{\Gamma}$ on the cylinder $Z$ over a 3-Sasakian manifold $M$ can be written as

$$
\tilde{\Gamma}=\tilde{\Gamma}^{i} I_{i}=e^{\mu} \tilde{\Gamma}_{\mu}^{i} I_{i}
$$

It is related with the Levi-Civita connection $\Gamma$ by formulae

$$
-\tilde{\Gamma}_{\mu \alpha}^{\nu}=\tilde{\Gamma}_{\mu \nu}^{\alpha}=\Gamma_{\mu \nu}^{\alpha}+3 P_{\alpha \mu \nu}, \quad \tilde{\Gamma}_{\mu a}^{b}=\Gamma_{\mu a}^{b},
$$

and has the torsion

$$
T^{\alpha}=3 P_{\alpha \mu \nu} e^{\mu \nu} \quad \text { and } \quad T^{a}=\frac{3}{2} P_{a \mu \nu} e^{\mu \nu}
$$

which is antisymmetric for the choice $\exp (2 h)=2$ in the metric.

\section{Instantons in higher dimensions}

\subsection{Reduction to matrix equations}

Recall that for all cases of manifolds $M$ considered in section 2 the instanton equation on the cone $\mathcal{C}(M)$ is equivalent to the equation

$$
* \mathcal{F}+* Q_{Z} \wedge \mathcal{F}=0
$$

on the cylinder $Z=\mathbb{R} \times M$ with the metric $g_{Z}=\mathrm{d} \tau^{2}+g_{M}$. The explicit form of the 4 -form $Q_{Z}$ on $Z$ was written down for all cases in section 2 . Let us denote by $G$ the holonomy group ${ }^{2}$ of the

\footnotetext{
${ }^{2}$ This holonomy group $G$ is the group $G_{2}, \operatorname{Spin}(7), \mathrm{SU}(m+1)$ and $\mathrm{Sp}(m+1)$ for cones over nearly Kähler, nearly parallel $G_{2}$, Sasaki-Einstein and 3-Sasakian manifolds $M$, respectively.
} 
Levi-Civita connection on $\mathcal{C}(M)$ and by $H$ the structure group ${ }^{3}$ of the canonical connection $\tilde{\Gamma}$ on $M$ (and also on $Z$ ). For the Lie algebras $\mathfrak{g}=\operatorname{Lie} G$ and $\mathfrak{h}=\operatorname{Lie} H$ we have

$$
\mathfrak{g}=\mathfrak{h} \oplus \mathfrak{m},
$$

where $\mathfrak{m}$ is an orthogonal complement of $\mathfrak{h}$ in $\mathfrak{g}$. Let $e^{0}=\mathrm{d} \tau$ and $e^{\mu}$ be an orthonormal basis of $T^{*} Z$. Then $e^{\mu}$ form a basis of $T^{*} M \subset T^{*} Z$ and their linear span can be identified with the vector space $\mathfrak{m}$.

Consider the generators

$$
\left[\hat{I}_{i}, \hat{I}_{j}\right]=f_{i j}^{k} \hat{I}_{k}, \quad\left[\hat{I}_{i}, \hat{I}_{\mu}\right]=f_{i \mu}^{\nu} \hat{I}_{\nu} \quad \text { and } \quad\left[\hat{I}_{\mu}, \hat{I}_{\nu}\right]=f_{\mu \nu}^{i} \hat{I}_{i}+f_{\mu \nu}^{\sigma} \hat{I}_{\sigma}
$$

acting on the space $V$ of an irreducible representation of $G$. These generators satisfy the same commutation relations as the generators $I_{i}, I_{a}$. In section 2 we wrote down the realization of these generators via the embedding $\mathfrak{g} \subset \mathfrak{s o}(n+1)$ with $n+1=\operatorname{dim} Z$ as acting (via infinitesimal rotations) on tangent spaces of $Z$. For this special representation we omit hats and note that $\tilde{\Gamma}=\tilde{\Gamma}^{i} I_{i}$ is the canonical connection whose curvature

$$
\tilde{R}=\mathrm{d} \tilde{\Gamma}+\tilde{\Gamma} \wedge \tilde{\Gamma}=\left(\mathrm{d} \tilde{\Gamma}^{i}+\frac{1}{2} f_{j k}^{i} \tilde{\Gamma}^{j} \wedge \tilde{\Gamma}^{k}\right) I_{i}
$$

satisfies the instanton equation (3.1) (see [1]).

Note that instead of tangent bundle $T Z$ one can consider an arbitrary vector bundle $\mathcal{V} \rightarrow Z$ with the structure group $G$ such that fibres are representations $V$ (real, complex or quaternionic) of the group $G$. For simplicity, we consider irreducible representations $V$ of the group $G$. The bundle $\mathcal{V} \rightarrow Z$ is associated with the principal bundle $P(Z, G)$. Since $H$ is a closed subgroup of $G$, it also acts on fibres of $\mathcal{V}$, but in general after restriction to $H \subset G$ the representation $V$ decomposes into a sum of irreducible representations $V_{q_{r}}$ of $H$ such that $V=\oplus_{r} V_{q_{r}}$. We denoted the generators of the group $G$ in the representation $V$ as $\hat{I}_{i}, \hat{I}_{\mu}$, where $\hat{I}_{i} \in \mathfrak{h}$ and $\hat{I}_{\mu} \in \mathfrak{m}$ for the splitting (3.2). Consider a connection

$$
\widehat{\Gamma}:=\tilde{\Gamma}^{i} \hat{I}_{i}
$$

on the bundle $\mathcal{V}$. In general, it is a reducible connection. Here $\tilde{\Gamma}^{i}$ are components of the canonical connection on the tangent bundle $T Z$. It is obvious that the curvature $\widehat{R}=\mathrm{d} \widehat{\Gamma}+\widehat{\Gamma} \wedge \widehat{\Gamma}$ of $\widehat{\Gamma}$ also satisfies the instanton equation (3.1).

Let us consider matrix-valued functions $X_{\mu}(\tau) \in \operatorname{End}(V)$ and introduce a connection

$$
\mathcal{A}:=\widehat{\Gamma}+X_{\mu} e^{\mu}
$$

on the vector bundle $\mathcal{V} \rightarrow Z$. Note that for matrices $X_{\mu}$ depending on all coordinates of $Z$, (3.6) is a general form of a connection on the bundle $\mathcal{V} \rightarrow Z$. For $X_{\mu}$ depending only on $\tau$, the instanton equation (3.1) will be reduced to ordinary differential equations on matrices $X_{\mu}$.

Recall that

$$
\mathrm{d} e^{\mu}=-\tilde{\Gamma}_{\nu}^{\mu} \wedge e^{\nu}+T^{\mu}=-\tilde{\Gamma}^{i} \wedge e^{\nu} f_{i \nu}^{\mu}+\frac{1}{2} T_{\sigma \nu}^{\mu} e^{\sigma} \wedge e^{\nu}
$$

\footnotetext{
${ }^{3}$ This structure group is the group $\mathrm{SU}(3), G_{2}, \mathrm{SU}(m)$ and $\mathrm{Sp}(m)$ for nearly Kähler, nearly parallel $G_{2}$, SasakiEinstein and 3-Sasakian manifolds, respectively.
} 
where $f_{i \nu}^{\mu}$ are structure constants from (3.3). From (3.6) and (3.7) it follows that

$$
\mathcal{F}=\mathrm{d} \mathcal{A}+\mathcal{A} \wedge \mathcal{A}=\mathrm{d} \widehat{\Gamma}+\widehat{\Gamma} \wedge \widehat{\Gamma}+\frac{1}{2}\left(\left[X_{\mu}, X_{\nu}\right]+T_{\mu \nu}^{\sigma} X_{\sigma}\right) e^{\mu} \wedge e^{\nu}+\dot{X}_{\nu} e^{0} \wedge e^{\nu}+\tilde{\Gamma}^{i} \wedge e^{\mu}\left(\left[\hat{I}_{i}, X_{\mu}\right]-f_{i \mu}^{\nu} X_{\nu}\right)
$$

where $\dot{X}_{\nu}=\frac{\mathrm{d} X_{\nu}}{\mathrm{d} \tau}$. Note that $\widehat{R}=\mathrm{d} \widehat{\Gamma}+\widehat{\Gamma} \wedge \widehat{\Gamma}$ satisfies to eq.(3.1) and $\mathcal{F}$ solves the instanton equation (3.1) on $Z$ if there are satisfied the following matrix equations:

$$
\begin{gathered}
{\left[\hat{I}_{i}, X_{\mu}\right]=f_{i \mu}^{\nu} X_{\nu}} \\
{\left[X_{\mu}, X_{\nu}\right]+T_{\mu \nu}^{\sigma} X_{\sigma}=N_{\mu \nu}^{\sigma} \dot{X}_{\sigma}+f_{\mu \nu}^{i} N_{i}(\tau) .}
\end{gathered}
$$

Here $N_{\mu \nu}^{\sigma}$ are some constants which we shall specify below for each case, $N_{i}$ are some $v(\mathfrak{h})$-valued functions defined by eqs.(3.10) after resolving the algebraic constraint equations (3.9) and substituting their solutions $X_{\mu}$ into (3.10). Here $v: \mathfrak{g} \rightarrow \operatorname{End}(V)$ is a representation of $\mathfrak{g}$. For $X_{\mu}$ satisfying (3.9)-(3.10), we have

$$
\mathcal{F}=\mathrm{d} \widehat{\Gamma}+\widehat{\Gamma} \wedge \widehat{\Gamma}+\frac{1}{2} N_{i} f_{\mu \nu}^{i} e^{\mu \nu}+\dot{X}_{\sigma}\left(e^{0 \sigma}+\frac{1}{2} N_{\mu \nu}^{\sigma} e^{\mu \nu}\right),
$$

where the term with $f_{\mu \nu}^{i}$ also satisfies (3.1) due to properties of $f_{\mu \nu}^{i}$ and the last term with $\dot{X}_{\sigma}$ satisfies (3.1) for choices of $N_{\mu \nu}^{\sigma}$ specified below for each considered case. Note that the constraint equations (3.9) for some examples of groups $G$ and $H$ were discussed and resolved e.g. in [25, 26] in the context of the equivariant dimensional reductions on coset spaces $G / H$. For special cases of the ansatz (3.6) instanton solutions were obtained e.g. in $[17,18,1]$. Peculiar property of such $\tau$-dependent solutions is that they can be lifted to gauge 5 -brane solutions of heterotic supergravity equations as was shown e.g in $[20,1,23]$.

\subsection{Reduction for nearly Kähler and nearly parallel $G_{2}$ manifolds}

Consider a manifold $M$ which is nearly Kähler $(\operatorname{dim} M=n=6)$ or nearly parallel $G_{2}(\operatorname{dim} M=$ $n=7)$. For both cases $\mu=a=1, \ldots, n$ with $n=6$ or $n=7$. Note that the 2 -forms

$$
e^{0 a}-\frac{1}{2 \rho} P_{a b c} e^{b c}
$$

solve the instanton equation (3.1) on $Z=\mathbb{R} \times M$ for $Q_{Z}$ and $P$ given in section 2 . Here $\rho=2$ for $n=6$ and $\rho=3$ for $n=7$. The generators $\hat{I}_{a}$ introduced in (3.3) are images of the 2-forms (3.12) under the metric-induced isomorphism $\Lambda^{2} Z \cong \mathfrak{s o}(7) \supset \mathfrak{g}_{2} \supset \mathfrak{m}$ for $n=6$ and $\Lambda^{2} Z \cong \mathfrak{s o}(8) \supset$ $\mathfrak{s p i n}(7) \supset \mathfrak{m}$ for $n=7$.

For both the nearly Kähler and nearly parallel $G_{2}$ cases we have

$$
T_{b c}^{a}=-f_{b c}^{a} \quad \text { and } \quad N_{b c}^{a}=\frac{1}{2} f_{b c}^{a}
$$

where $N_{b c}^{a}$ are defined by comparing the components of $\mathcal{F}$ in (3.11) and the explicit form (3.12) of (parts of) anti-self-dual forms on $Z$. Thus, we obtain the following matrix equations:

$$
\begin{gathered}
{\left[\hat{I}_{i}, X_{a}\right]=f_{i a}^{b} X_{b}} \\
{\left[X_{a}, X_{b}\right]=f_{a b}^{c}\left(X_{c}+\frac{1}{2} \dot{X}_{c}\right)+f_{a b}^{i} N_{i}(\tau) .}
\end{gathered}
$$


Substituting the ansatz $X_{a}=\phi \hat{I}_{a}$ with a real function $\phi(\tau)$, we see that (3.14) are satisfied and (3.15) are reduced to the equation

$$
\dot{\phi}=2 \phi(\phi-1)
$$

obtained in [1] and for $N_{i}$ we obtain $N_{i}=\phi^{2} \hat{I}_{i}$. More general equations can be obtained by choosing more general solution of the constraint equations (3.14). Such solutions for different choice of groups $G$ and $H$ were discussed e.g. in [25, 26]. Constructing solutions to eqs. (3.9), (3.10) goes beyond the scope of this short article. This task will be considered elsewhere.

\subsection{Reduction for Sasaki-Einstein manifolds}

Consider $Z=\mathbb{R} \times M$ with a Sasaki-Einstein manifold $M$. In this case $\mu=(1, a)$ with $a=2, \ldots, 2 m+1$ and the 2 -forms

$$
e^{01}-\frac{1}{m+1} \omega_{a b} e^{a b} \quad \text { and } \quad \exp (h)\left(e^{0 a}+\omega_{a b} e^{1 b}\right)
$$

solve the instanton equations (3.1) with $Q_{Z}$ given in (2.24). From (2.23), (3.8) and (3.17) we obtain

$$
T_{a b}^{1}=-f_{a b}^{1}, \quad T_{1 b}^{a}=-f_{1 b}^{a}, \quad N_{a b}^{1}=\frac{1}{m+1} f_{a b}^{1} \quad \text { and } \quad N_{1 b}^{a}=\frac{m}{m+1} f_{1 b}^{a} .
$$

Substituting (3.18) into (3.9)-(3.10), we obtain

$$
\begin{gathered}
{\left[\hat{I}_{i}, X_{1}\right]=0, \quad\left[\hat{I}_{i}, X_{a}\right]=f_{i a}^{b} X_{b},} \\
{\left[X_{1}, X_{a}\right]=f_{1 a}^{b}\left(X_{b}+\frac{m}{m+1} \dot{X}_{b}\right) .} \\
{\left[X_{a}, X_{b}\right]=f_{a b}^{1}\left(X_{1}+\frac{1}{m+1} \dot{X}_{1}\right)+f_{a b}^{i} N_{i}(\tau) .}
\end{gathered}
$$

If we choose $X_{1}=\chi \hat{I}_{1}$ and $X_{a}=\psi \hat{I}_{a}$ then (3.19) are satisfied, for $N_{i}$ we obtain $N_{i}=\psi^{2} \hat{I}_{i}$ and (3.20)-(3.21) are reduced to the equations

$$
\dot{\chi}=(m+1)\left(\psi^{2}-\chi\right) \quad \text { and } \quad \dot{\psi}=\frac{m+1}{m} \psi(\chi-1)
$$

coinciding with those obtained in [1]. Note that (3.22) is a gradient flow equation

$$
\dot{x}^{i}=g^{i j} \frac{\partial W}{\partial x^{j}} \quad \text { for } \quad W=\left(x^{1}\right)^{2}-2 x^{1}\left(x^{2}\right)^{2}+2\left(x^{2}\right)^{2}-1, i, j=1,2,
$$

for the metric on $\mathbb{R}^{2}$ of the form

$$
\mathrm{d} s^{2}=g_{i j} \mathrm{~d} x^{i} \mathrm{~d} x^{j}=\frac{2}{m+1}\left(\mathrm{~d} x^{1}\right)^{2}+\frac{4 m}{m+1}\left(\mathrm{~d} x^{2}\right)^{2}
$$

with $x^{1}:=\chi$ and $x^{2}:=\psi[1]$. 


\subsection{Reduction for 3-Sasakian manifolds}

For $Z=\mathbb{R} \times M$ with a 3 -Sasakian manifold $M$ we have $\mu=(\alpha, a), \alpha=1,2,3$ and $a=4, \ldots, 4 m+3$. One can check that the image of the generators $\hat{I}_{a}$ from (3.3) under the map into $\Lambda^{2} Z \cong \mathfrak{s o}(4 m+4) \supset$ $\mathfrak{s p}(m+1) \supset \mathfrak{m}$ is given by the 2 -forms

$$
e^{0 \alpha}-\frac{1}{3} \varepsilon_{\alpha \beta \gamma} e^{\beta \gamma} \text { and } \exp (h)\left(e^{0 a}+\omega_{a b}^{\alpha} e^{\alpha b}\right)
$$

which satisfy to eq.(3.1) with $Q_{Z}$ given in (2.32). From (2.30), (2.35), (3.8) and (3.23) it follows that

$$
\begin{gathered}
T_{a b}^{\alpha}=-\frac{3}{2} f_{a b}^{\alpha}, \quad T_{a \beta}^{b}=f_{a \beta}^{b}, \quad T_{\beta \gamma}^{\alpha}=-f_{\beta \gamma}^{\alpha}, \\
N_{a \beta}^{b}=f_{a \beta}^{b} \quad \text { and } \quad N_{\beta \gamma}^{\alpha}=\frac{1}{2} f_{\beta \gamma}^{\alpha}
\end{gathered}
$$

with other components vanishing. Substituting (3.24)-(3.25) into (3.9)-(3.10), we obtain

$$
\begin{gathered}
{\left[\hat{I}_{i}, X_{\alpha}\right]=0, \quad\left[\hat{I}_{i}, X_{a}\right]=f_{i a}^{b} X_{b}} \\
{\left[X_{\alpha}, X_{\beta}\right]=f_{\alpha \beta}^{\gamma}\left(X_{\gamma}+\frac{1}{2} \dot{X}_{\gamma}\right), \quad\left[X_{a}, X_{\beta}\right]=f_{a \beta}^{b}\left(X_{b}+\dot{X}_{b}\right)} \\
{\left[X_{a}, X_{b}\right]=f_{a b}^{\alpha} X_{\alpha}+f_{a b}^{i} N_{i}(\tau) .}
\end{gathered}
$$

If we choose the ansatz $X_{\alpha}=\chi \hat{I}_{\alpha}$ and $X_{a}=\psi \hat{I}_{a}$ then (3.26) will be satisfied identically, from (3.28) we obtain $N_{i}=\psi^{2} \hat{I}_{i}$ and (3.27)-(3.28) reduce to the equations

$$
\dot{\chi}=2 \chi(\chi-1), \quad \dot{\psi}=\psi(\chi-1) \quad \text { and } \quad \chi=\psi^{2},
$$

where the last algebraic equation follows from (3.28). These equations coincide with those obtained in [1]. Thus, our ansatz (3.6) which leads to matrix equations (3.9)-(3.10) generalizes the "scalar" ansatz of the paper [1] and allows one to obtain more general instanton solutions. However, obtaining explicit instanton solutions lies beyond the scope of our paper.

\section{Generalizations: quiver bundles}

\subsection{Smaller groups $H$}

Recall that we considered nearly Kähler, nearly parallel $G_{2}$, Sasaki-Einstein and 3-Sasakian manifolds $M$ with the structure groups $\mathrm{SU}(3), G_{2}, \mathrm{SU}(m)$ and $\mathrm{Sp}(m)$, respectively, following to Harland and Nölle who considered in their ansatz [1] exactly the above groups with generators in the defining vector representation of the group $\mathrm{SO}(n+1) \supset H$, i.e. $V=\mathbb{R}^{n+1}$ with $n=6,7,2 m+1$ and $4 m+3, m=1,2, \ldots$. However, the group $H$ can be smaller than the above-mentioned Lie groups, i.e. often $H$ lies inside the group $\mathrm{SU}(3), G_{2}, \mathrm{SU}(m)$ and $\mathrm{Sp}(m)$, respectively. In this case, the constraint equations (3.9) become weaker and allow more degrees of freedom in matrices $X_{a}$. For instance, for the nearly Kähler coset space

$$
M=\mathrm{SU}(3) / \mathrm{U}(1) \times \mathrm{U}(1)
$$


the structure group is $H=\mathrm{U}(1) \times \mathrm{U}(1)$ that increase the number of functions parameterizing the ansatz (3.6) even for vector representation $V=\mathbb{R}^{7} \cong \mathbb{R} \oplus \mathbb{C}^{3}$ with $\mathfrak{g}_{2} \supset \mathfrak{s u}(3)=\mathfrak{u}(1) \oplus \mathfrak{u}(1) \oplus \mathfrak{m}$. Writing the ansatz (3.6) in terms of $\mathfrak{s u ( 3 )}$-valued matrices $X_{a}$, one can resolve (3.9) as

$$
\begin{aligned}
X_{1}=\left(\begin{array}{ccc}
0 & 0 & -\phi_{1} \\
0 & 0 & 0 \\
\bar{\phi}_{1} & 0 & 0
\end{array}\right), X_{3}=\left(\begin{array}{ccc}
0 & -\bar{\phi}_{2} & 0 \\
\phi_{2} & 0 & 0 \\
0 & 0 & 0
\end{array}\right), X_{5}=\left(\begin{array}{ccc}
0 & 0 & 0 \\
0 & 0 & -\bar{\phi}_{3} \\
0 & \phi_{3} & 0
\end{array}\right), \\
X_{2}=\left(\begin{array}{ccc}
0 & 0 & \mathrm{i} \phi_{1} \\
0 & 0 & 0 \\
\mathrm{i} \bar{\phi}_{1} & 0 & 0
\end{array}\right), X_{4}=-\left(\begin{array}{ccc}
0 & \mathrm{i} \bar{\phi}_{2} & 0 \\
\mathrm{i} \phi_{2} & 0 & 0 \\
0 & 0 & 0
\end{array}\right), X_{6}=-\left(\begin{array}{ccc}
0 & 0 & 0 \\
0 & 0 & \mathrm{i} \bar{\phi}_{3} \\
0 & \mathrm{i} \phi_{3} & 0
\end{array}\right),
\end{aligned}
$$

where $\phi_{1}, \phi_{2}, \phi_{3}$ are complex-valued functions of $\tau$ and the generators $I_{7,8}$ of the subgroup $\mathrm{U}(1) \times \mathrm{U}(1)$ of $\mathrm{SU}(3)$ are chosen in the form

$$
I_{7}=-\mathrm{i}\left(\begin{array}{ccc}
0 & 0 & 0 \\
0 & 1 & 0 \\
0 & 0 & -1
\end{array}\right) \quad \text { and } \quad I_{8}=\frac{\mathrm{i}}{\sqrt{3}}\left(\begin{array}{ccc}
2 & 0 & 0 \\
0 & -1 & 0 \\
0 & 0 & -1
\end{array}\right) .
$$

Substituting (4.2) into (3.15), we obtain equations

$$
\dot{\phi}_{1}=-2 \phi_{1}+2 \bar{\phi}_{2} \bar{\phi}_{3}, \quad \dot{\phi}_{2}=-2 \phi_{2}+2 \bar{\phi}_{1} \bar{\phi}_{3}, \quad \dot{\phi}_{3}=-2 \phi_{3}+2 \bar{\phi}_{1} \bar{\phi}_{2}
$$

and constraints

$$
N_{7}=\Phi I_{7}, \quad N_{8}=-\sqrt{3} \Phi I_{8} \quad \text { with } \quad \Phi=\phi_{1} \bar{\phi}_{1}=\phi_{2} \bar{\phi}_{2}=\phi_{3} \bar{\phi}_{3}
$$

for a proper normalization of the structure constants. From (4.5) we see that complex-valued functions $\phi_{1}, \phi_{2}, \phi_{3}$ can differ only in their phase parts. For $\phi_{1}=\phi_{2}=\phi_{3}=: \phi$ eqs.(4.4) reduce to eq.(3.16) on a real-valued function $\phi$.

\subsection{Reducible representations of $H$ and quiver bundles}

Similar situation takes place for nearly parallel $G_{2}$-manifolds, where as an example one can consider the Aloff-Wallach space $\mathrm{SU}(3) / \mathrm{U}(1)$ with the structure group $H=\mathrm{U}(1)$ (see the second paper in [18] for discussion of solving eqs.(3.14)), and also for Sasaki-Einstein and 3-Sasakian manifolds the structure group $H$ can be a closed subgroup of $\mathrm{SU}(m)$ and $\mathrm{Sp}(m)$, respectively. Even more freedom appears if one considers an irreducible representation $V$ of the holonomy group $G$ of the cone $\mathcal{C}(M)$ which decomposes into a sum of irreducible representations $V_{q_{r}}$ of the group $H$,

$$
V=\bigoplus_{r=1}^{\ell} V_{q_{r}} \quad \text { with } \quad \sum_{r=1}^{\ell} q_{r}=q
$$

so that

$$
\hat{I}_{i}=\left(\begin{array}{cccc}
I_{i}^{q_{1}} & 0 & \ldots & 0 \\
0 & \ddots & & \vdots \\
\vdots & & \ddots & 0 \\
0 & \ldots & 0 & I_{i}^{q_{\ell}}
\end{array}\right)
$$


Here $I_{i}^{q_{r}}$ are generators of $q_{r} \times q_{r}$ irreducible representations $V_{q_{r}}$ of $H$ and $V \cong \mathbb{C}^{q}$ (or $\mathbb{R}^{q}$ or $\mathbb{H}^{q} \cong \mathbb{R}^{4 q}$ ). If we assume that $H$ contains a maximal abelian subgroup of $G$ then the remaining generators $\hat{I}_{a}$ of $G$ in this representation have the off-diagonal form ${ }^{4}$

$$
\hat{I}_{a}=\left(\begin{array}{cccc}
0 & I_{a}^{q_{12}} & \ldots & I_{a}^{q_{1 \ell}} \\
I_{a}^{q_{21}} & 0 & \ddots & \vdots \\
\vdots & \ddots & \ddots & I_{a}^{q_{\ell-1 \ell}} \\
I_{a}^{q_{\ell 1}} & \ldots & I_{a}^{q_{\ell \ell-1}} & 0
\end{array}\right)
$$

where $I_{a}^{q_{r s}}$ are $q_{r} \times q_{s}$ matrices (cf. [12]).

Thus, one can associate a bounded quiver ${ }^{5}$ satisfying a set of relations $R$ to the ansatz (3.6) for a connection on a vector bundle $\mathcal{V} \rightarrow \mathcal{C}(M)$ over nearly Kähler, nearly parallel $G_{2}$, Sasaki-Einstein and 3-Sasakian manifolds. In the simplest case of generators (4.7) the matrices $X_{a}$ solving the constraint equations are obtained from (4.8) by substituting $\phi_{r s} I_{a}^{q_{r s}}$ instead of $I_{a}^{q_{r s}}$, where $\phi_{r s}$ are complex functions of $\tau$. Important fact is that the space $\mathcal{C}(M)$ is not homogeneous and therefore quivers and quiver bundles can appear in dimensional reduction without $G$-equivariance condition studied earlier e.g. in $[25,26]$. Recall that another way in which quiver gauge theories arise as low-energy effective field theories in string theory is through considering cones and orbifolds with conical singularities and placing D-branes at the orbifold singularities [28]. Our constructions can be lifted as in [23] to heterotic strings and provide a description of NS5-branes and gauge NS5branes. It would be of interest to study further this brane interpretation and its possible relations with constructions of [28].

\subsection{Kähler-Einstein manifolds and quiver gauge theories}

As another example related with quiver gauge theories we consider the manifold

$$
\mathcal{Y}=\Sigma \times \mathcal{X}
$$

where $\Sigma$ and $\mathcal{X}$ are 2-dimensional and $2 k$-dimensional Kähler-Einstein manifolds with the Kähler form $\omega$ on $\Sigma$ and $\Omega$ on $\mathcal{X}$. Let $\widehat{\Gamma}$ be the canonical $\mathfrak{u}(k)$-valued Levi-Civita connection on $\mathcal{X}$,

$$
\widehat{\Gamma}=\Gamma^{i} \hat{I}_{i} \quad \text { with } \quad \hat{I}_{i} \in \mathfrak{u}(k) .
$$

We consider $\mathrm{U}(k)$ as a closed subgroup of the Lie group $\mathrm{SU}(k+1)$. Let $V \cong \mathbb{C}^{q}$ be an irreducible representation of the group $\mathrm{SU}(k+1)$ decomposed into a sum of irreducible representations $V_{q_{r}} \cong$ $\mathbb{C}^{q_{r}}$ of the group $\mathrm{U}(k)$ as in (4.7) and $\mathcal{V} \rightarrow \mathcal{X}$ is a holomorphic vector bundle over $\mathcal{X}$ associated with the bundle $P(\mathcal{X}, \mathrm{U}(k))$ of hermitian frames on $\mathcal{X}$. This bundle has the connection (4.10) which is reducible according to $(4.7), \mathcal{V}=\oplus_{r} \mathcal{V}_{q_{r}}$.

\footnotetext{
${ }^{4}$ If $H$ does not contain a maximal abelian subgroup of $G$ or there is a subgroup in $G$ commuting with $H$ then $\hat{I}_{a}$ in (4.8) will contain diagonal terms $I_{a}^{q_{r r}}$ with $r=1, \ldots, \ell$.

${ }^{5}$ A quiver $Q=\left(Q^{0}, Q^{1}\right)$ is an oriented graph, i.e. a set of vertices $Q^{0}$ with a set $Q^{1}$ of arrows between the vertices (see e.g. [27]). A path in $Q$ is a sequence of arrows in $Q^{1}$ which compose. A relation of the quiver is a formal finite sum of paths. In our case vertices correspond to vector bundles $\mathcal{V}_{q_{r}}$ with fibres $V_{q_{r}}$ and arrows correspond to morphisms $\mathcal{V}_{q_{s}} \rightarrow \mathcal{V}_{q_{r}}$ of vector bundles.
} 
Consider now $\ell$ complex vector bundles $E_{1}, \ldots, E_{\ell}$ over $\Sigma$ with unitary connections $A^{1}, \ldots, A^{\ell}$ and ranks $N_{1}, \ldots, N_{\ell}$. Introduce a complex vector bundle $\mathcal{E}=\oplus_{r} E_{r} \otimes \mathcal{V}_{q_{r}}$ over $\Sigma \times \mathcal{X}$ of rank

$$
N=\sum_{r=1}^{\ell} N_{r} q_{r}
$$

and assume $c_{1}(\mathcal{E})=0$ without loss of generality, so that the structure group of $\mathcal{E}$ is $\mathrm{SU}(N)$. The matrices

$$
\tilde{I}_{i}:=\left(\begin{array}{cccc}
\mathbf{1}_{N_{1}} \otimes I_{i}^{q_{1}} & 0 & \ldots & 0 \\
0 & \ddots & & \vdots \\
\vdots & & \ddots & 0 \\
0 & \ldots & 0 & \mathbf{1}_{N_{\ell}} \otimes I_{i}^{q_{\ell}}
\end{array}\right)
$$

are generators of a reducible unitary representation of the group $\mathrm{U}(k)$ on the complex vector space $\widetilde{V} \cong \mathbb{C}^{N}$. Introduce a gauge connection

$$
A:=\left(\begin{array}{cccc}
A^{1} \otimes \mathbf{1}_{q_{1}} & 0 & \ldots & 0 \\
0 & \ddots & & \vdots \\
\vdots & & \ddots & 0 \\
0 & \ldots & 0 & A^{\ell} \otimes \mathbf{1}_{q_{\ell}}
\end{array}\right)
$$

on the bundle $E:=\oplus_{r} E_{r} \otimes \mathbb{C}^{q_{r}}$ over $\Sigma$. It is obvious from (4.13) that $\left[A, \tilde{I}_{i}\right]=0$.

On the bundle $\mathcal{E} \rightarrow \mathcal{Y}$ we introduce a connection

$$
\mathcal{A}=A+\Gamma^{i} \tilde{I}_{i}+X_{a} e^{a}
$$

where $X_{a} \in \mathfrak{s u}(N)$ are matrices which depend only on coordinates of $\Sigma$ and $e^{a}$ is the basis of 1 -forms on $\mathcal{X}, a=1, \ldots, 2 k$. Note that

$$
\mathrm{d} e^{a}=-\Gamma_{b}^{a} \wedge e^{b}=-f_{i b}^{a} \Gamma^{i} \wedge e^{b} .
$$

Using (4.15), for the curvature $\mathcal{F}$ of the connection (4.14) we obtain

$$
\mathcal{F}=\mathrm{d} \mathcal{A}+\mathcal{A} \wedge \mathcal{A}=F+\widetilde{R}+\left(\mathrm{d} X_{a}+\left[A, X_{a}\right]\right) \wedge e^{a}+\frac{1}{2}\left[X_{a}, X_{b}\right] e^{a} \wedge e^{b}+\left(\left[I_{i}, X_{a}\right]-f_{i a}^{b} X_{b}\right) \Gamma^{i} \wedge e^{a},
$$

where

$$
F=\mathrm{d} A+A \wedge A, \quad \widetilde{R}=\mathrm{d} \widetilde{\Gamma}+\widetilde{\Gamma} \wedge \widetilde{\Gamma} \quad \text { and } \quad \widetilde{\Gamma}:=\Gamma^{i} \tilde{I}_{i}
$$

Suppose that $X_{a}$ satisfy the constraints

$$
\left[\tilde{I}_{i}, X_{a}\right]=f_{i a}^{b} X_{b}
$$

and impose on $\mathcal{F}$ the Hermitian-Yang-Mills equations [7]

$$
\begin{gathered}
\mathcal{F}^{0,2}=0 \quad \Rightarrow \quad \bar{\partial} X_{a}+\left[A^{0,1}, X_{a}\right]=0, \quad\left[Y_{\bar{A}}, Y_{\bar{B}}\right]=0, \\
(\omega+\Omega)\lrcorner \mathcal{F}=0 \quad \Rightarrow \quad \omega^{\alpha \beta} \mathcal{F}_{\alpha \beta}+\lambda \tilde{I}_{0}+\Omega^{a b}\left[X_{a}, X_{b}\right]=0 .
\end{gathered}
$$


Here $\bar{\partial}+A^{0,1}$ is the anti-holomorphic part of the covariant derivative on $\Sigma$,

$$
Y_{\overline{1}}:=\frac{1}{2}\left(X_{1}+\mathrm{i} X_{k+1}\right), \ldots, Y_{\bar{k}}:=\frac{1}{2}\left(X_{k}+\mathrm{i} X_{2 k}\right),
$$

the constant $\lambda$ is proportional to the scalar curvature of the Kähler-Einstein manifold $\mathcal{X}, \omega_{\alpha \beta}$ and $\Omega_{a b}$ are components of the Kähler forms on $\Sigma$ and $\mathcal{X}, \tilde{I}_{0}$ is the $\mathfrak{u}(1)$ generator in the decomposition $\mathfrak{u}(k)=\mathfrak{u}(1) \oplus \mathfrak{s u}(k) \subset \mathfrak{s u}(k+1), \alpha, \beta=1,2$ and $A, B, \ldots=1, \ldots, k$. We see that (4.19)-(4.20) are the usual quiver vortex equations on $\Sigma(\operatorname{cf} .[12,26]) .{ }^{6}$ For $k=1$ and $\mathcal{X}=\mathbb{C} P^{1}$, one can obtain [29] the standard vortex equations on a Riemann surface $\Sigma$. One can generalize the above construction by taking instead of $\Sigma$ a Kähler-Einstein manifold of dimension more than two.

\subsection{On instantons on smooth manifolds}

It is of interest to extend the ansatz for a connection $\mathcal{A}$ from cones to their smooth resolutions as it was proposed in [20] as well as from direct product manifolds, such as $\mathcal{Y}$ in section 4.3, to irreducible smooth manifolds with warped product metrics. This is possible.

For illustration we consider non-compact Calabi-Yau $(k+1)$-folds $\mathcal{Z}$ discussed in [20]. They have a metric

$$
\mathrm{d} \tilde{s}^{2}=\frac{\mathrm{d} r^{2}}{f^{2}(r)}+r^{2} f^{2}(r) \eta^{2}+2 r^{2} \mathrm{~d} s_{K E}^{2}
$$

where

$$
f^{2}=1-\left(\frac{a^{2}}{r^{2}}\right)^{k+1}
$$

$\mathrm{d} s_{K E}^{2}$ is the standard Kähler metric on a Kähler-Einstein manifold $\mathcal{X}$ which is the base manifold for a projection

$$
\pi: \mathcal{X}^{\prime} \rightarrow \mathcal{X}
$$

from Sasaki-Einstein $(2 k+1)$-manifold $\mathcal{X}^{\prime}$ onto $\mathcal{X}$ and $\eta$ is the 1 -form along fibres of the projection (4.23).

Note that

$$
\mathrm{d} \tilde{s}^{2}=r^{2} \mathrm{~d} s^{2}
$$

with

$$
\mathrm{d} s^{2}=\frac{\mathrm{d} r^{2}}{r^{2} f^{2}}+f^{2} \eta^{2}+2 \mathrm{~d} s_{K E}^{2}=\frac{\mathrm{d} \tau^{2}}{f^{2}}+f^{2} \eta^{2}+2 \mathrm{~d} s_{K E}^{2},
$$

i.e. $\mathrm{d} \tilde{s}^{2}$ is conformally equivalent to $\mathrm{d} s^{2}$. Singularity of the transformation at $r=0$ is not essential since we are interested in Yang-Mills instantons on the manifold with the metric (4.21) extendable smoothly at $r=0$. Note also that the instanton equation on $\mathcal{Z}$ is invariant w.r.t. conformal transformation,

$$
\tilde{*} F+\tilde{*} Q_{Z} \wedge F=\tilde{*} F+(\tilde{\omega}+\tilde{\Omega})^{k-1} \wedge F=r^{2(k-1)}\left(* F+(\omega+\Omega)^{k-1} \wedge F\right)=0,
$$

since

$$
\tilde{\omega}+\tilde{\Omega}=r^{2}(\omega+\Omega)
$$

\footnotetext{
${ }^{6}$ Note that the last equations in (4.19) correspond to quiver relations.
} 
and $\tilde{*}=r^{2(k-1)} *$. Here $\omega=\mathrm{d} \tau \wedge \eta$ and $\Omega$ is the Kähler form on $\mathcal{X} \hookrightarrow \mathcal{Z}$.

Ansatz for $\mathcal{A}$ on the space $\mathcal{Z}^{\prime}$ with the metric (4.25) is the same as in (4.14) and lead to the same reduction (4.19)-(4.20) of the instanton equation. Solving these vortex equations, one obtains instantons on $\mathcal{Z}$. One can simplify the task assuming that $\mathcal{A}_{\eta}$ and $X_{a}$ depend only on $\tau=\ln r$ and choosing $\mathcal{A}_{\tau}=0$. Then (4.19)-(4.20) will be reduced to equations similar to those which were considered in $[20]$.

\section{Conclusions}

We have examined in some detail the construction of instantons on cones $\mathcal{C}(M)$ over nearly Kähler and nearly parallel $G_{2}$-manifolds $M$ initiated in $[17,18]$ and extended to cones over Sasaki-Einstein and 3-Sasakian manifolds in $[1,20]$. Having at our disposal a reduced structure group $H$ of a manifold $M$ admitting real Killing spinors and the holonomy group $G$ of the cone $\mathcal{C}(M)$, we introduced a quiver bundle $\mathcal{V}$ over $\mathcal{C}(M)$, determined entirely by the representation theory of the group $G$ and $H$, and introduced a proper connection $\mathcal{A}$ on this bundle. The ansatz for $\mathcal{A}$ reduces the instanton equations on $\mathcal{C}(M)$ to simpler matrix equations which can be solved in many special cases. It is of interest to construct new instanton solutions on $\mathcal{C}(M)$ by using our generalized ansatz, and to lift them to solutions of heterotic supergravity along the way considered in [1, 23].

We have also introduced a quiver bundle $\mathcal{E}$ over a Kähler-Einstein manifold of the form $\Sigma \times \mathcal{X}$ and extended the Levi-Civita connection on the $2 k$-dimensional Kähler-Einstein manifold $\mathcal{X}$ to a connection on $\mathcal{E}$ parameterized by $2 k$ matrices $X_{a}$. We established an equivalence between solutions of Hermitian-Yang-Mills equations on $\Sigma \times \mathcal{X}$ and solutions of some quiver vortex equations on $\Sigma$.

Recall that regular Sasaki-Einstein manifolds are U(1)-bundles over Kähler-Einstein manifolds and cones over them are Calabi-Yau spaces. Using this correspondence, we have introduced a connection on a quiver bundle $\mathcal{E}$ over smooth resolutions of $(2 k+2)$-dimensional Calabi-Yau cones. It is of interest to consider instantons on other special holonomy manifolds. ${ }^{7}$ We hope to report on this in the future.

\section{Acknowledgements}

The authors thank the Institute for Theoretical Physics of Hannover University, where this work was completed, for hospitality. This work was partially supported by the Deutsche Forschungsgemeinschaft, the Russian Foundation for Basic Research and the Heisenberg-Landau program.

\footnotetext{
${ }^{7}$ Some instanton solutions on particular kinds of $G_{2^{-}}$and $\operatorname{Spin}(7)$-manifolds were considered in [30].
} 


\section{References}

[1] D. Harland and C. Nölle, "Instantons and Killing spinors," JHEP 03 (2012) 082 [arXiv:1109.3552 [hep-th]].

[2] A.A. Belavin, A.M. Polyakov, A.S. Schwartz and Yu.S. Tyupkin, "Pseudoparticle solutions of the Yang-Mills equations," Phys. Lett. B 59 (1975) 85.

[3] T. Eguchi, P.B. Gilkey and A.J. Hanson, "Gravitation, gauge theories and differential geometry," Phys. Rept. 66 (1980) 213.

[4] R. Rajaraman, Solitons and Instantons, North-Holland, Amsterdam, 1984.

[5] E. Corrigan, C. Devchand, D.B. Fairlie and J. Nuyts, "First order equations for gauge fields in spaces of dimension greater than four," Nucl. Phys. B 214 (1983) 452.

[6] R.S. Ward, "Completely solvable gauge field equations in dimension greater than four," Nucl. Phys. B 236 (1984) 381.

[7] S.K. Donaldson, "Anti-self-dual Yang-Mills connections on a complex algebraic surface and stable vector bundles," Proc. Lond. Math. Soc. 50 (1985) 1;

S.K. Donaldson, "Infinite determinants, stable bundles and curvature," Duke Math. J. 54 (1987) 231;

K.K. Uhlenbeck and S.-T. Yau, "On the existence of Hermitian-Yang-Mills connections on stable bundles over compact Kähler manifolds," Commun. Pure Appl. Math. 39 (1986) 257.

[8] M. Mamone Capria and S.M. Salamon, "Yang-Mills fields on quaternionic spaces,"

Nonlinearity 1 (1988) 517;

R. Reyes Carrión, "A generalization of the notion of instanton," Diff. Geom. Appl. 8 (1998) 1.

[9] L. Baulieu, H. Kanno and I.M. Singer, "Special quantum field theories in eight and other dimensions," Commun. Math. Phys. 194 (1998) 149 [arXiv:hep-th/9704167];

M. Blau and G. Thompson, "Euclidean SYM theories by time reduction and special holonomy manifolds," Phys. Lett. B 415 (1997) 242 [hep-th/9706225];

B.S. Acharya, J.M. Figueroa-O'Farrill, B.J. Spence and M. O'Loughlin, "Euclidean D-branes and higher-dimensional gauge theory," Nucl. Phys. B 514 (1998) 583 [arXiv:hep-th/9707118].

[10] S.K. Donaldson and R.P. Thomas, "Gauge theory in higher dimensions,"

in: The Geometric Universe, Oxford University Press, Oxford, 1998;

S.K. Donaldson and E. Segal, "Gauge theory in higher dimensions II", arXiv:0902.3239 [math.DG].

[11] G. Tian, "Gauge theory and calibrated geometry,"

Ann. Math. 151 (2000) 193 [arXiv:math/0010015 [math.DG]].

[12] A.D. Popov, "Non-Abelian vortices, super-Yang-Mills theory and Spin(7)-instantons,"

Lett. Math. Phys. 92 (2010) 253 [arXiv:0908.3055 [hep-th]];

A.D. Popov and R.J. Szabo, "Double quiver gauge theory and nearly Kähler flux compactifications," JHEP 02 (2012) 033 [arXiv:1009.3208 [hep-th]]. 
[13] M.B. Green, J.H. Schwarz and E. Witten, Superstring theory, Cambridge University Press, Cambridge, 1987.

[14] K. Becker, M. Becker and J.H. Schwarz, String theory and M-theory: A modern introduction, Cambridge University Press, Cambridge, 2007.

[15] D.B. Fairlie and J. Nuyts, "Spherically symmetric solutions of gauge theories in eight dimensions," J. Phys. A 17 (1984) 2867;

S. Fubini and H. Nicolai, "The octonionic instanton," Phys. Lett. B 155 (1985) 369;

A.D. Popov, "Anti-self-dual solutions of the Yang-Mills equations in $4 n$-dimensions," Mod. Phys. Lett. A 7 (1992) 2077;

T.A. Ivanova and A.D. Popov, "(Anti)self-dual gauge fields in dimension $d \geq 4$," Theor. Math. Phys. 94 (1993) 225.

[16] E. Corrigan, P. Goddard and A. Kent, "Some comments on the ADHM construction in 4kdimensions," Commun. Math. Phys. 100 (1985) 1;

E.K. Loginov, "Multi-instantons in higher dimensions and superstring solitons," SIGMA 1 (2005) 002 [arXiv:hep-th/0511262];

J. Broedel, T.A. Ivanova and O. Lechtenfeld, "Construction of noncommutative instantons in $4 k$ dimensions," Mod. Phys. Lett. A 23 (2008) 179 [arXiv:hep-th/0703009].

[17] T.A. Ivanova, O. Lechtenfeld, A.D. Popov and T. Rahn, "Instantons and Yang-Mills flows on coset spaces," Lett. Math. Phys. 89 (2009) 231 [arXiv:0904.0654 [hep-th]];

D. Harland, T.A. Ivanova, O. Lechtenfeld and A.D. Popov, "Yang-Mills flows on nearly Kähler manifolds and $G_{2}$-instantons," Commun. Math. Phys. 300 (2010) 185 [arXiv:0909.2730 [hep-th]];

D. Harland and A.D. Popov, "Yang-Mills fields in flux compactifications on homogeneous manifolds with SU(4)-structure," JHEP 02 (2012) 107 [arXiv:1005.2837 [hep-th]].

[18] I. Bauer, T.A. Ivanova, O. Lechtenfeld and F. Lubbe, "Yang-Mills instantons and dyons on homogeneous $G_{2}$-manifolds," JHEP 10 (2010) 044 [arXiv:1006.2388 [hep-th]];

A.S. Haupt, T.A. Ivanova, O. Lechtenfeld and A.D. Popov, "Chern-Simons flows on Aloff-Wallach spaces and Spin(7)-instantons,"

Phys. Rev. D 83 (2011) 105028, [arXiv:1104.5231 [hep-th]];

K.-P. Gemmer, O. Lechtenfeld, C. Nölle and A.D. Popov, "Yang-Mills instantons on cones and sine-cones over nearly Kähler manifolds," JHEP 09 (2011) 103 [arXiv:1108.3951 [hep-th]].

[19] C. Bär, "Real Killing spinors and holonomy," Commun. Math. Phys. 154 (1993) 509.

[20] F.P. Correia, "Hermitian Yang-Mills instantons on Calabi-Yau cones," JHEP 12 (2009) 004 [arXiv:0910.1096 [hep-th]];

F.P. Correia, "Hermitian Yang-Mills instantons on resolutions of Calabi-Yau cones," JHEP 02 (2011) 054 [arXiv:1009.0526 [hep-th]]. 
[21] A. Strominger, "Heterotic solitons," Nucl. Phys. B 343 (1990) 167;

J.A. Harvey and A. Strominger, "Octonionic superstring solitons,"

Phys. Rev. Lett. 66 (1991) 549;

T.A. Ivanova, "Octonions, self-duality and strings," Phys. Lett. B 315 (1993) 277;

M. Gunaydin and H. Nicolai, "Seven-dimensional octonionic Yang-Mills instanton and its extension to an heterotic string soliton," Phys. Lett. B 351 (1995) 169 [arXiv:hep-th/9502009].

[22] O. Lechtenfeld, C. Nölle and A.D. Popov, "Heterotic compactifications on nearly Kähler manifolds," JHEP 09 (2010) 074 [arXiv:1007.0236 [hep-th]];

C. Nölle, "Homogeneous heterotic supergravity solutions with linear dilaton,"

J. Phys. A 45 (2012) 045402 [arXiv:1011.2873 [hep-th]];

A. Chatzistavrakidis, O. Lechtenfeld and A. D. Popov, "Nearly Kähler heterotic compactifications with fermion condensates," arXiv:1202.1278 [hep-th].

[23] K.-P. Gemmer, A.S. Haupt, O. Lechtenfeld, C. Nölle and A.D. Popov, "Heterotic string plus five-brane systems with asymptotic $\mathrm{AdS}_{3}, "$ arXiv:1202.5046 [hep-th].

[24] C.P. Boyer and K. Galicki, "Sasakian geometry, holonomy, and supersymmetry," arXiv:math/0703231.

[25] O. Garcia-Prada, "Invariant connections and vortices," Commun. Math. Phys. 156 (1993) 527;

L. Alvarez-Consul and O. Garcia-Prada, "Dimensional reduction and quiver bundles," J. Reine Angew. Math. 556 (2003) 1 [arXiv:math/0112160].

[26] A.D. Popov and R.J. Szabo, "Quiver gauge theory of nonabelian vortices and noncommutative instantons in higher dimensions," J. Math. Phys. 47 (2006) 012306 [arXiv:hep-th/0504025];

O. Lechtenfeld, A. D. Popov and R. J. Szabo, "Quiver gauge theory and noncommutative vortices," Prog. Theor. Phys. Suppl. 171 (2007) 258 [arXiv:0706.0979 [hep-th]];

O. Lechtenfeld, A.D. Popov and R.J. Szabo, "SU(3)-equivariant quiver gauge theories and nonabelian vortices," JHEP 08 (2008) 093 [arXiv:0806.2791 [hep-th]];

B.P. Dolan and R.J. Szabo, "Dimensional reduction and vacuum structure of quiver gauge theory," JHEP 08 (2009) 038 [arXiv:0905.4899 [hep-th]].

[27] M. Auslander, I. Reiten and S.O. Smalø, Representation theory of Artin algebras, Cambridge University Press, Cambridge, 1995.

[28] M.R. Douglas and G.W. Moore, "D-branes, quivers and ALE instantons," hep-th/9603167;

C.V. Johnson and R.C. Myers, "Aspects of Type IIB theory on ALE spaces,"

Phys. Rev. D 55 (1997) 6382 [hep-th/9610140];

M.R. Douglas, B. Fiol and C. Romelsberger, "The spectrum of BPS branes on a noncompact Calabi-Yau," JHEP 09 (2005) 057 [hep-th/0003263].

[29] A.D. Popov, "Integrability of vortex equations on Riemann surfaces,"

Nucl. Phys. B 821 (2009) 452 [arXiv:0712.1756 [hep-th]]; 
A.D. Popov, "Non-abelian vortices on Riemann surfaces: an integrable case," Lett. Math. Phys. 84 (2008) 139 [arXiv:0801.0808 [hep-th]].

[30] S. Miyagi, "Yang-Mills instantons on 7-dimensional manifold of G(2) holonomy," Mod. Phys. Lett. A 14 (1999) 2595 [arXiv:hep-th/9911184];

H. Kanno and Y. Yasui, "Octonionic Yang-Mills instanton on quaternionic line bundle of Spin(7) holonomy," J. Geom. Phys. 34 (2000) 302 [arXiv:hep-th/9910003];

M. Dunajski and M. Hoegner, "SU(2) solutions to self-duality equations in eight dimensions," arXiv:1109.4537 [hep-th]. 